\title{
Téoros
}

Revue de recherche en tourisme

\section{Connaissances en tourisme et reconnaissance sociale}

\section{Jean Stafford}

Volume 11, numéro 1, mars 1992

La formation en tourisme : à la croisée des chemins

URI : https://id.erudit.org/iderudit/1078951ar

DOI : https://doi.org/10.7202/1078951ar

Aller au sommaire du numéro

Éditeur(s)

Université du Québec à Montréal

ISSN

0712-8657 (imprimé)

1923-2705 (numérique)

Découvrir la revue

Citer cet article

Stafford, J. (1992). Connaissances en tourisme et reconnaissance sociale.

Téoros, 11(1), 44-46. https://doi.org/10.7202/1078951ar d'utilisation que vous pouvez consulter en ligne.

https://apropos.erudit.org/fr/usagers/politique-dutilisation/ 


\section{Connaissances en tourisme et reconnaissance sociale}

Nous voulons aborder ici le problème de la formation en tourisme àpartir de l'état des connaissances propres a ce domaine. C'est un truisme d"affirmer que l'université est chargée de produire et d'enseigner des connaissances mais, en matière de tourisme, la validité et l'utilité de ces connaissances sont loin de faire l'unanimité. Le statut scientifique des connaissanoes touristiques et la reconnaissance sociale des diplồmes et des professions du tourisme sont étroitement reliés.

Nous allons donc toucher successivement les points suivants:

- les niveaux des connaissances touristiques:

- $1^{+}$objet d'étude du tourisme;

- les processus de différenciation;

- connaissances théoriques et connaissances pratiques;

- la validité des connaissances:

- les connaissances touristiques dans la société (la reconnaissance sociale).

\section{Les niveaux des connaissances touristiques}

On peut assez facilement tracer un tableau des niveaux des connaissances propres au domaine touristique. On peut imaginer cinq niveaux;

A- des images, des impressions, des sentiments, des perceptions liés aux vacances et aux voyages;

B- des connaissances journalistiques: celles véhiculées dans les médias;

$\mathrm{C}$ - des connaissances générales: les descriptions des voyages de Stendhal ou de Proust;

D- des connaissances particulières qui touchent une profession ou un métier: manipulation d' un terminal de réservation informatique, recettes culinaires régionales, etc.+

E- des connaissancesscientifiques; ils'agit de connaissances spécifiques orientées vers l'élaboration de théories, d'hypothèses fonctionnelles ou tendancielles, de lois et de règles générales.
Seules les connaissances scientifiques (le niveau E) peuvent être généralisables parce qu'elles sont construites àdes fins précises et de façon rigoureuse. L'imagerie commune suppose des chemins de passage d'un niveau à un autre mais, comme l'a si bien montré Gaston Bachelard, cette vision des choses est fausse. Les connaissances scientifiques s'établissent en opposition, en dépit et malgre les autres niveaux des connaissances.

Les connaissances scientifiques du tourisme devront, pour se constituer, faire la distinction et la critique des autres niveaux des connaissances. Comme le souligne Gaston Bachelard:

... l'objectivité se détermine dans la précision et dans la cohérence des attributs, non pas dans la collection des objets plus ou moins analogues. Cela est si vrai que ce qui limite une connaissance est. souvent plus important, pour les progrès de lapensée, que ce qui étend vaguement la connaissance $e^{(1)}$.

\section{L'objet d'étude du tourisme}

Il existe beaucoup de définitions du tourisme et aucune n'est vraiment satisfaisante. Déjà en 1972, René Baretje et Pierre Defert écrivaient:

Cecaractère insaisissablen'est pas propre au tourisme. Nombreux sont les secteurs de la connaissance quisont d'autant moins saisis convenablement par notre esprit que l'analyse y fait apparaître sans cesse des arrière-plans de plus en plus étendus ${ }^{(2)}$.

Malgré tout, ce problème d'identification demeure vital pour le domaine du tourisme.

L'objet d'étude du tourisme pourrait être defini à partir des fonctions remplies par les connaissances propres à ce secteur. On peut penser qu'il y a connaissances touristiques 
lorsque l'on tente d'intégrer des éléments nombreux et variés qui touchent les vacances et les voyages. Par exemple, nous croyons qu' $1 n^{4} y$ a pas de système de comptabilité, ni de méthodes de gestion de personnel qui soient proprement touristiques. Le tourisme n'existe, n'apparaît que dans une approche multifonctionnelle. Cette démarche correspond semble-t-il à la fois, à la structure de l'offre et de la demande ${ }^{(3)}$ touristiques.

L'objet d'étude du tourisme peut aussi être cerné à partir des problemes particuliers que posent les recherches touristiques ${ }^{(4)}$; ces problèmes sont une illustration pratique de la specificité des connaissances en matière de tourisme. Un autre argument, que l'on peut emprunter à l'épistémologie des sciences de la gestion ${ }^{(3)}$, est le fait que la téorologie (les connaissances scicntifiques du tourisme) n'est étudiée, de façon systématique, par aucune autre discipline etque son absence (du champ des sciences) serait une perte et un obstacle important, à moyen et à long terme, au développement de l'industrie touristique.

\section{Les processus de différenciation}

En s'élaborant, les connaissances touristiques établissent des mécanismes de différenciation. Ces mécanismes vont jouer à deux niveaux différents:

... une diffêrenciation interne qui distingue les différentes disciplines entre elles, et une différenciation externe qui établit une coupure entre une discipline scientifique et les aspects extra-scientifiques de la culture générale ${ }^{\dagger}$.

On a vu, dans les niveaux des connaissances touristiques, qu'il est relativement facile de distinguer les connaissances scientifiques en tourisme des connaissances générales ou journalistiques. Les différences entre la téorologie (la connaissance du tourisme) et les autres disciplines sont plus difficiles a cemer.

Le principal point de différence entre la térologie et les disciplines connexes est la nécessité d'une approchemultifonctionnelle propre à son objet d'étude: cette nécessité objective d'animer des paliers de réalité differents et complexes. La téorologie se définira par cette capacité d'intégrer des éléments disparates liés à l'espace et au temps, à l'économie et à la politique, à la psycholo- gie et à la gestion et enfin, à la culture et au patrimoine.

Il semble que la distinction entre la téorologie et les autres disciplines se fera pair petites étapes et sera surtout causée par le désintérêt que provoque le champ touristique dans les disciplines traditionnelles. Cette vision des choses est nettement perceptible en 6conomie, en sociologie, en histoireet, à un moindre degré, en géographie. Les sociologues et les 6conomistes (etles autres aistes:), spécialistes de la téorologie, sont toujours percus comme des atouristes: à l'intérieur de leur propre discipline. L'indépendance de la téorologie se fera donc par défaut et grâce à la formulation de programmes de recherche intégrant les multiples facettes du tourisme.

La téorologie dans les sciences de la gestion connait des problemes similaires. Pour Elie Cohen: «Les connaissances de gestion constituent un ensemble disparate dont toutes les composantes ne peuvent revendiquer le même statut épistemologiquew $n$. Les frontières entre certaines des disciplines des sciences de la gestion sont parfois nébuleuses. On retrouvera cette difficulte en gestion touristique; par exemple, une distinction claire entre le marketing des services et le marketing touristique n'est pas facile à faire au niveau des concepts et des méthodes. La, comme dans les sciences sociales, les connaissances touristiques entrent en jeu quand on fait appel à une approche «transversales qui intègre des éléments nombreux et diversifies tels que:

- le marché touristique;

- lescomportements des consommateurs de voyage:

- l'évolution des loisirs touristiques;

- des éléments liés a l'espace et a la localisation;

- des valeurs propres au tourisme et aux loisirs, etc.

\section{Connaissances théoriques et connaissances pratiques}

Ilestdemise, dans certainsmilieux, $\mathrm{d}^{4}$ opposer constamment connaissances theoriques et connaissances pratiques. Les connaissances théoriques ne seraient que ades idées en l'airs, l'activité principale de spelleteux de nuages", $d$ 'incorrigibles rêveurs! Les connaissances pratiques feraient partie du monde réel, de l'incontournable quotidienneté. Pourtant, dans la réalité, les choses sont bien différentes: dans l'élaboration du moindre projet touristique on se heurte, de façon im- placable, à l'absence de données valables, a l'utilisation de méthodes rudimentaires et peu fiables. On peut faire l'hypothèse que la faiblesse actuelle de l'industrie touristique est due, en grande partie, à cette faiblesse theorique.

Un fait touristique est une donnce pratique (utilisable) formulée à partir d'une réflexion théorique: la théorie, même implicite, est préalable à l'observation et a l'ctablissement des faits. Pour Alan Chalmers:
La science ne commence pas par des énoncés d'observation parce qu'il faut une théorie avant tout énoncé d'observa- tion, et les énoncés d'obser- vation, parce qu'ils sont failli- bles, ne constituent pas une base sûre sur laquelle la con- naissance scientifique peutetre fondé $e^{(3)}$.

Les faits scientifiques sont construits par une théorie; il est vrai que cette théorie peut être minimaleouimplicite ${ }^{(9)}$. Selon Carl Hempel:
... lamaxime selon laquelle on doit rassembler les données sans être guidée par une hy- pothèse antérieure sur les re- lations entre les faits que l'on étudie se détruit elle-même, et personne ne la suit dans une recherche scientifique ${ }^{(10)}$.

Par exemple, en tourisme au Québec, la notion de congrès a été utilisée de façon très lăche; une premiere définition en faisait un véritable fourre-tout, elle incluait les réunions. d'organisation des clubs de hockey locaux, les mariages, etc. $\mathrm{L}^{*} \mathrm{ABCQ}$ ( $\mathrm{I}^{*}$ Association des bureaux de congrèsduQuébec) estarrivé, à partir d"unecertaine theorisation du tourisme de congres, à une définition plus stricte et plus opérationnelle. Ainsi, lacomptablisation d'un congrès doit répondre à certains criteres:

1- cinquante personnes ou plus;

2- membres d'une association reconnue;

3- sejour d'une durée supérieure à 24 heures (une nuitee ou plus);

4 dont l'objet doit être d'échanger des idées ou se communiquer des études sur des sujets d'intérêts communs.

Cette nouvelle définition (d'ailleurs perfectible) est moins criticable. Elle apparaît comme la construction d'une informa- 
tion spécialisée susceptible d'être utilisable dans des hypothèses et des modeles de la réalitétudiée. La connaissance scientifique en tourisme vise donc à déterminer des eléments spécifiques et particuliers qui s'opposent aux connaissances communes. Malheureusement, une bonne partie du champ touristique semble encore un vaste bric-âbrac géré par des démiurges ${ }^{(1)}$.

\section{La validité des connaissances en tourisme}

PourElie Cohen, la validitédes connaissances

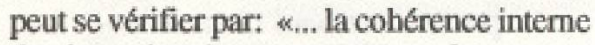
des énoncés et leur capacité à rendre compte du comportement réel des entreprises, avec un certain degré de généralitéw ${ }^{(12)}$. Les critères pour distinguer les connaissances scientifiques des connaissancesnon scientifiques peuvent être multiples. Ainsi, la qualité des problèmes à aborder, la force des hypothèses et la capacité des théories à régler, en tout ou enpartie, cesproblèmessontaussides critères importants.

Pour Karl Popper, le développement des théories se faiten fonction du schémasuivant:

$\mathrm{P} 1 \rightarrow \mathrm{TT} \rightarrow \mathrm{EE} \rightarrow \mathrm{P} 2 ;$

où:

P1 et P2 = problème traité avant et après;

TT = est la théorie à l'essai;

$\mathrm{EE}=$ discussion critique de $\mathrm{l}^{\mathrm{T}}$ erreur ${ }^{(13)}$.

Le progres des connaissances survient dans la distance entre P1 et $\mathrm{P} 2$; ce qui reste du problème à étudier.

Les conséquences pour la téorologie sont les suivantes:

- la téorologie doit établir (avec l'industrie touristique) une liste graduée des problèmes majeurs;

- elledoittenterd'élaborerdes hypothèses portant sur ces problemes;

- elle doit enfin faire une critique systématique des résultats obtenus. Ces critiques auront des répercussions sur les hypothèses et les méthodologies employées.

Cette approche critique est essentielle. Pour Karl Popper: «...l'hypothèse la mieux adaptée est celle qui résout le mieux le problème qu'elle avait pour fonction de résoudre, et celle qui résiste le mieux à la critique que les hypothèses concurrentes ${ }^{(14)}$. Cette conception de l'hypothèse va à l'encontre de la croyance populaire qui cherche, dans les théories, une impossible synthèse, un amalgame hétéroclite alors que la connaissance objective naît de la confrontation des idées. Cette vision des choses rend légitime l'existence de paradigmes concurrents ${ }^{(15)}$.

Pour la téorologie, qui est une science embryonnaire, la philosophie poppérienne pourrait facilement être adaptée ${ }^{(16)}$. Il n'en reste pas moins que la démarche décrite plus haut sembleirreversible. Comme le souligne Jean-Marie Gagnon, pour les sciences de la gestion:

... le sort des sciences de l'administration est irréversiblement liế à celui des autres disciplines universitaires. Leur méthodologie évoluera dans la même mesure et la même direction que celle de leurs voisines ${ }^{(17)}$.

La téorologie (au niveau de l'université) ne pourra echapper à cette lourde influence. Dans le futur, ce contrôle de la communauté scientifique va s'alourdir et la validité scientifique des connaissances deviendra la principale mesure des performances.

D' ici l'an 2000, pour l' établissement de connaissances scientifiques dans le domaine du tourisme, d'enormes investissements seront nécessaires. Les techniques pratiques de l'ingénieur s'appuient sur les théories de la physique et des mathématiques. De la même façon, l'étudiant, le praticien en tourisme doiventdisposer $d^{4}$ un corps deconnaissances rigoureux justifiant leurs expertises et leurs pratiques de gestion et d'intervention.

\section{Les connaissances touristiques dans la société}

C'est un leurre de croire que la visibilité sociale des étudiants et des praticiens en tourisme pourra se faire en faisant l'économie d'un corps de connaissances scientifiques sur le tourisme. Une reconnaissance sociale durable passera par le biais de la reconnaissance scientifique. Lareconnaissance sociale des professions du tourisme (de la formation en tourisme) ne peut être qu'une question d'image; elle doit être aussi une question de fond! Il faut pouvoir user d'un corpus minimal de problèmes importants, d'hypothèses plausibles et de théories généralisables. Nos diplômés devraient posséder des outils d'analyse et des moyens efficaces pour faire face aux besoins des industries touristiques.
En outre, au plan sociologique, ces connaissances scientifiques devraient assurer une plus grande cohésion des différents champs du domaine touristique. Elles fourniraient un vocabulaire commun, des approches et des méthodologies communes. Les problèmes, les théories et les hypothèses vont ainsi jouer le rôle d' un «cordon sanitaires entre les connaissances scientifiques propres au tourisme et les autres connaissances.

Je suis pessimiste pour le court terme et optimiste pour le long terme. Les problèmes de la formation touristique et de sa reconnaissance sociale ne sont pas insolubles.

Les solutions apparaissent dans la création de programmes de formation et de recherche $^{\text {(18) }}$ qui intègrent le multiples facettes du tourisme dans une démarche multi-fonctionnelle, respectueuse des différences et rigoureuse dans sa formulation.

\section{NOTES ET RÉFÉRENCES}

(1) G. Bachelard, La formation de Iresprit secientifique, Vrin, Paris, 1967, p. 71

(2) R. Baretje et P. Defert, Aipects 6 conomiques du touriame, Berger-Levrault, Paris, 1972, p. 17.

(3) Voir a ce sujet G. Gay-Para, La pratique du tourisme, Économica, Paris, 1985 .

(4) Voir d ce sujet B.J. Fitchie, Some Critical Aspects of Messurement Theory and Practice in Travel Research, Journal of Travel Research. wol. 14. no 1. 1975

(5) Voir à ce sujet E. Cohen, Epistémologie de la gestion, Encyclopédle de gestion, tome 1, Economica, Paris, 1989

(6) M. Leclerc, Introduction al I'btude socio-politique des sciences, Département de science politique. Note de recherche no 18, UOAM, 1980 . politiquer.
p. 53 .

(7) E. Cohen, op, eit., p, 1058

(B) A. Chalmers, Ou'est-ce que la selence? Editions La Decouverte, Paris, 1987, p. 54.

(9) Voir à ce suiet J.L. Le Moigne, Epistémologies constructivistes et sciences de forganisation, dans A. Marinet (et autres), Epistémologies ot scienA. Marinet (et autres), Episténologies at

(10) C. Hempel, Eláments d'bpistómologie, A. Colin, Paris, 1972, p. 19.

(11) Les difficultés récentes du groupe Malenfant illustrent cette situation.

(12) E. Cohen, op, cit. p. 1073.

(13) K. Popper, La connaissance objective, Aubier Paris, 1991, pp. 427-428.

(14) K. Popper, op. cit., p. 395.

(15) Vair aे ce sujet $J$, Staiford, Les paradigmes de la recherche en tébologie: étude, anakes et critque, Loisire et société, wol. 8, no 2, Trois-Aividres, 1965 .

(16) Voir à ce sujet J. Girin. Analyse empirique des situations de gestion: elements de thérie ef de méthode, dans A. Martinet (et autres), Epistémologie et sciences de gestion. Economica, Paris. 1990, pp. 171-178.

(17) J.-M. Gagnon, La demarche inteliectuelle: une note sur le cas des ecoles unversitities d'administration, dans M. Audet et J.-L. Malouin (et autres). La production des connaissances selentifiques do Iradministration, Les Presses de IUniversite Laval, Quebec, 1966, p. 62.

(18) En ce sens, la création du Centre international de formation et de recherche en tourisme ICIFORT] et de la Chaire en tourisme a l'UOAM sont des pas dans la bonne direction. 